\title{
Clinical Efficacy of Imeglimin (Twymeeg) for Elderly Patient with Type 2 Diabetes Mellitus (T2DM)
}

\author{
Masaki OKADA ${ }^{1}$, Hiroshi BANDO ${ }^{1,2,3^{*}}$, Noboru IWATSUKI ${ }^{1}$, Tomoya OGAWA ${ }^{1}$, Kazuki SAKAMOTO ${ }^{1}$ \\ ${ }^{1}$ Sakamoto Hospital, Higashi Kagawa city, Kagawa, Japan \\ ${ }^{2}$ Tokushima University/Medical Research, Tokushima, Japan \\ ${ }^{3}$ Japan Low Carbohydrate Diet Promotion Association, Kyoto, Japan
}

Corresponding Author: Hiroshi BANDO, MD, PhD, FACP ORCID iD

Address: Tokushima University /Medical Research, Nakashowa 1-61, Tokushima 770-0943, Japan; Email: pianomed@bronze.ocn.ne.jp

Received date: 16 January 2022; Accepted date: 14 February 2022; Published date: 21 February 2022

Citation: Okada M, Bando H, Iwatsuki N, Ogawa T, Sakamoto K. Clinical Efficacy of Imeglimin (Twymeeg) for Elderly Patient with Type 2 Diabetes Mellitus (T2DM). Asp Biomed Clin Case Rep. 2022 Feb 21;5(1):33-37.

Copyright (C) 2022 Okada M, Bando H, Iwatsuki N, Ogawa T, Sakamoto K. This is an open-access article distributed under the Creative Commons Attribution License, which permits unrestricted use, distribution, and reproduction in any medium provided the original work is properly cited.

\section{Abstract}

Background: As an oral hypoglycaemic agent (OHA), imeglimin (Twymeeg) has been recently introduced to clinical practice for patients with type 2 diabetes mellitus (T2DM) as Twymeeg. It has beneficial pharmacological mechanisms, which are improving insulin secretion, increasing insulin sensitivity and decreasing insulin resistance.

Case Presentation: The case is 84-year-old men with mild cognitive impairment (MCI) for 3 years. He visited late August, 2021 our clinic for general malaise and was pointed out to have post-prandial blood glucose 336 $\mathrm{mg} / \mathrm{dL}$ and HbA1c 8.6\%. He was diagnosed as T2DM.

Results: He was started to be given imeglimin 10oomg twice a day, and then HbA1c value was decreased to $7.3 \%$ in 4 weeks and 5.7\% in 8 weeks. During 9-12 weeks, he felt loss of appetite and reduced food intake. Biochemical examination on 12 weeks showed decreased values of TP, Alb, HbA1c, glucose, free $\mathrm{T}_{3}$ and normal values of TSH, free $\mathrm{T}_{4}$. Doses of imeglimin were $500 \mathrm{mg}$ twice a day for 9-12 weeks and discontinued after 12 weeks.

Discussion: Regarding appetite loss, possible causes may include MCI, previous history of gallbladder dyskinesia, adverse effect of imeglimin, and so on. Further development of research will be expected for imeglimin in the future.

\section{Keywords}

Oral Hypoglycemic Agent, Imeglimin, Twymeeg, Type 2 Diabetes Mellitus, American Diabetes Association

\section{Abbreviations}

OHA: Oral Hypoglycemic Agent (OHA); T2DM: Type 2 Diabetes Mellitus; ADA: American Diabetes Association

\section{Introduction}

Adequate treatment for type 2 diabetes mellitus (T2DM) has been discussed for long. Professional practice committee of American Diabetes Association
(ADA) has announced the guideline in Jan 2022 [1]. International Diabetes Federation (IDF) has managed diabetic situation in the world. When observing the diabetic situation across the world, the prevalence has 
been progressively increasing [2]. IDF has stated that about half adults (20-79 years) with diabetes do not know their diabetic situation [3]. DM has been a chronic and multifaced disease leading to significant physical detriment to many patients. During 2019, there were 463 million diabetic patients present across the world, and among them 90\% are suffering from T2DM [4]. The adequate medical treatment would be fit for each patient from ideal medical point of view.

Regarding pharmacological treatment for T2DM, metformin has been a widely used medication for T2DM despite the introduction of some newer antidiabetic agents. Metformin is one of the most used oral hypoglycemic agents (OHAs), and it has desirable pharmacokinetics. Recently, imeglimin was produced for OHA. It has basically a small molecule similar to metformin, but it has a cyclic molecule containing a triazine ring [5]. For its characteristics, it has beneficial pharmacological mechanisms. They are improving insulin secretion, increasing insulin sensitivity and decreasing insulin resistance [6]. It has been introduced to actual medical practice for patients with $\mathrm{T} 2 \mathrm{DM}$ as the brand name Twymeeg. In this report, an older patient with T2DM will be presented and discussed who has taken Twymeeg with clinical efficacy.

\section{Case Presentation}

History \& Physical:

The patient is an 84-year-old male patient with Type 2 Diabetes Mellitus (T2DM). He was diagnosed to have mild cognitive impairment (MCI) about 3 years ago. In March 2021, he felt fatigue and was found to have some exacerbation of MCI. He had been treated in another clinic. When he visited our clinic in August, 2021, he received general blood laboratory examination. The results showed moderate degree of diabetes with post-prandial blood glucose $336 \mathrm{mg} / \mathrm{dL}$ and HbA1c $8.6 \%$.

\section{Laboratory Examinations:}

The biochemical results of September 2021 were summarized as follows: AST $17 \mathrm{U} / \mathrm{L}$, ALT $23 \mathrm{U} / \mathrm{L}, \mathrm{\gamma}-\mathrm{GT}$ 20 U/L, ALP 154 U/L (38-113), LDH 159 U/L (124-222), T-Bil 1.6 mg/dL, TP 8.0 g/dL, Alb 3.8 g/dL, CPK 109 U/L (30-200), BUN $25 \mathrm{mg} / \mathrm{dL}, \mathrm{Cr} 0.8 \mathrm{mg} / \mathrm{dL}$, UA 4.2
mg/dL, Na 144 mEq/L, K 3.2 mEq/L, Cl 107 mEq/L, TC $149 \mathrm{mg} / \mathrm{dL}$ 、HDL-C $38 \mathrm{mg} / \mathrm{dL}$, LDL-C $89 \mathrm{mg} / \mathrm{dL}$, TG $109 \mathrm{mg} / \mathrm{dL}$. RBC $4.26 \times 10^{6} / \mu \mathrm{L}, \mathrm{Hb} 13.9 \mathrm{~g} / \mathrm{dL}, \mathrm{Ht}$ 41.9\%, MCV 98.4 fL (80-98), MCH 32.7 pg (27-34), MCHC 33.3 g/dL (31-36), WBC 810o / $\mu \mathrm{L}$, Plt $16.6 \times 10^{4}$ $/ \mu \mathrm{L}$. Chest X-P showed normal for lung and heart, and ECG was within normal limits.

\section{Clinical Progress}

He was diagnosed as T2DM. For diabetic treatment, he and his family received nutrition therapy for adequate regular meal. His age has been rather higher, then he was advised to have slight limited intake of carbohydrate amount per day. It was about $40 \%$ of calorie ratio of total meal a day. For diabetic medication, he was started to be provided Imeglimin (Twymeeg R) $1000 \mathrm{mg}$ twice in the morning and evening (Fig-1). After 4 weeks, blood glucose improved to $225 \mathrm{mg} / \mathrm{dL}$ and HbA1c $7 \cdot 3 \%$, and the blood glucose improved to $131 \mathrm{mg} / \mathrm{dL}$ and HbA1c 5.7\% after 8 weeks. From 9 weeks, imeglimin was decreased from 10oomg $\mathrm{x} 2$ to 50omg $\mathrm{x} 2$. The case had normal renal function for $\mathrm{Cr} 0.8 \mathrm{mg} / \mathrm{dL}$ and eGFR $69.4 \mathrm{~mL} / \mathrm{min} / 1.73 \mathrm{~m}^{2}$.

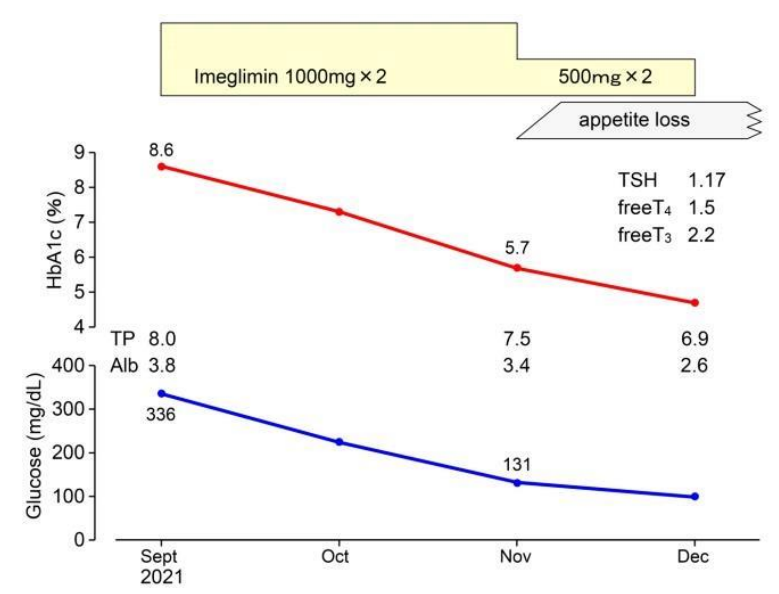

Fig-1: Clinical progress of the case for exams and medication

During 9-12 weeks, the patient had loss of appetite and reduced food intake. Regarding this status, unremarkable causes related to this situation were detected or suggested. Biochemical examination was conducted at the point of 12 weeks. TP and Alb on $\mathrm{O}$ week vs 12 weeks were $8.0 \mathrm{~g} / \mathrm{dL}$ vs $6.9 \mathrm{~g} / \mathrm{dL}, 3.8 \mathrm{~g} / \mathrm{dL}$ vs $2.6 \mathrm{~g} / \mathrm{dL}$, respectively. Diabetic and endocrinological exams on 12 weeks included HbA1c $4.7 \%$, blood glucose $100 \mathrm{mg} / \mathrm{dL}$, TSH $1.17 \mu \mathrm{IU} / \mathrm{ml}$ (o.5-5.o), free $\mathrm{T}_{4}$ 
$1.5 \mathrm{ng} / \mathrm{mL}(0.9-1.7)$, free $\mathrm{T}_{3} 2.2 \mathrm{pg} / \mathrm{mL}(2.3-4 \cdot 3)$. Due to the biochemical exams on 12 weeks, Twymeeg was discontinued, and glucose variability has been followed up after that.

\section{Ethical Considerations}

This study has been basically conducted along with the ethical principles on the Declaration of Helsinki. Moreover, several comments were obtained from Ethical Guidelines for Research for Humans, which are associated with Good Clinical Practice (GCP). The author and collaborators related to this study have established an ethical committee. It is in our hospital, including the president and director of the hospital, associated with physician, pharmacist, nurse, nutritionist and the legal professional. We have discussed enough and performed for adequate manners, and decided to present the agreements for this study protocol. The informed consent associated with written style of the agreement document were taken from the patient.

\section{Discussion}

In this report, a new OHA, imeglimin was administered to T2DM patient, which showed beneficial clinical effect. It seems to be a novel category of OHA, because it has multiple mechanisms for one medication. Clinical efficacy includes improved insulin secretion and decreased insulin resistance for peripheral tissue. It showed HbA1c reduction of about $0.5-1.0 \%$ by simple administration of $2000 \mathrm{mg} /$ day [7]. Furthermore, add-on therapy (AOT) reveals additional efficacy, where HbA1c was 0.6-0.65\% more decreased in the case of dipeptidyl peptidase- 4 inhibitor (DPP4i) agents [8]. Regarding adverse effects of imeglimin, no remarkable major reports were observed, such as various cardiovascular events, or increased hypoglycemia and others [6].

What is the fundamental characteristic mechanism for imeglimin? It has been the first medicine for tetrahydrotriazine-containing class agent as glimins [9]. It may work by multiple pathway mechanism such as increasing insulin secretion, improving beta-cell function and preventing epithelial cell death [10]). The detail mechanism is not fully understood, however it may work through the enhancement of glucose- stimulated insulin secretion (GSIS). This GSIS process includes activated transient receptor potential melastatin 2 (TRPM2) channels. As a result, it can promote the plasma membrane depolarization, which is one of non-selective cation channels (NSCCs) of beta-cells [11]. Moreover, imeglimin can be involved in the calcium mobilization for the amplification pathway during insulin secretion process [12].

In this case, administration of imeglimin reduced HbA1c enough to satisfactory level until 8 weeks. This degree of improvement is comparable to previous reports [6]. During 9-12 weeks, appetite loss had developed associated with the decrease in total protein, albumin, HbA1c and blood glucose values. For thyroid function, serum TSH and free $\mathrm{T}_{4}$ were within normal range, and free $\mathrm{T}_{3}$ showed slightly decreased, which suggested the situation of low $\mathrm{T}_{3}$ syndrome. This physiology has been often found in the elderly people, and no significant changes were observed in his endocrine and metabolic functions.

What could be the cause of loss of appetite in his clinical course? The case has suffered from some problems of mild cognitive impairment (MCI) or dementia. Then, it is possible that MCI caused a change in appetite. In addition, a previous history of dyskinesia in the gallbladder was found from detail history-taking. However, no abdominal pain or other abdominal symptoms were found in recent period. Another possibility would be from the adverse effect of imeglimin for GI symptoms [13]. However, there was no significant change in his symptoms during o-8 weeks, and the problem started after $9^{\text {th }}$ weeks. Consequently, it is not likely to be involved.

For Imeglimin, there is a report concerning treatment-emergent adverse events (TEAE). When imeglimin 500, 1000 or $1500 \mathrm{mg}$ and placebo is provided twice a day, the ratio observing any symptom and/or sign would be $68.0 \%, 62.2 \%, 73.3 \%$ and $68.0 \%$, respectively [6]. Slight increase of gastrointestinal (GI) adverse effects would be observed with the $1500 \mathrm{mg}$ dose level. Hypoglycemia was found in similar percentage for these groups. Consequently, imeglimin was well tolerated and beneficial for improving glucose variability without significant increase of hypoglycemic events compared with 
placebo. From some reports concerning 100omg vs 150omg, the former shows fewer events for GI problems.

Imeglimin possibly becomes a first-in-class agent for T2DM, which mainly shows excretion unchanged through the kidneys. The pharmacokinetic (PK) characteristics were investigated for the optimal amounts of doses in T2DM with chronic kidney disease (CKD) [14]. The recommended amount calculated from area under the curve (AUC) was 50omg provided twice morning and evening for patient with eGFR 15$45 \mathrm{~mL} / \mathrm{min} / 1.73 \mathrm{~m}^{2}$. Similary, 100omg of imeglimin taken twice daily seems to be optimal for patient with eGFR > $45 \mathrm{~mL} / \mathrm{min} / 1.73 \mathrm{~m}^{2}$. Additional study will be expected for larger doses of $1500 \mathrm{mg}$ twice daily in AUC method or eGFR value.

For clinical efficacy of imeglimin, meta-analysis study was conducted [7]. Data are from 8 studies including 1555 cases. Compared with control group, imeglimin group had the superior results for $\mathrm{HbA1c}$ and blood glucose. In contrast, there were not significant differences on HDL, LDL, triglyceride and HOMA-IR. Various researches will be required concerning monotherapy and combined therapy for imeglimin [13].

In summary, T2DM case with successful efficacy of imeglimin (Twymeeg) was described in this article. The mechanism of this agent has dual aspects, and it may become a new category of diabetic OHAs. Further development of diabetic practice and research will be expected for imeglimin and related matters in the future.

\section{Conflict of Interest}

The authors have read and approved the final version of the manuscript. The authors have no conflicts of interest to declare.

\section{Funding}

There was no funding received for this paper.

\section{References}

[1] American Diabetes Association Professional
Practice Committee; American Diabetes Association Professional Practice Committee:, Draznin B, Aroda VR, Bakris G, Benson G, Brown FM, Freeman R, Green J, Huang E, Isaacs D, Kahan S, Leon J, Lyons SK, Peters AL, Prahalad P, Reusch JEB, Young-Hyman D, Das S, Kosiborod M. 9. Pharmacologic Approaches to Glycemic Treatment: Standards of Medical Care in Diabetes-2022. Diabetes Care. 2022 Jan 1;45(Supplement_1):S125-S143. [PMID: 34964831]

[2] Aschner P, Karuranga S, James S, Simmons D, Basit A, Shaw JE, Wild SH, Ogurtsova K, Saeedi P; International Diabetes Federation's Diabetes Epidemiological Guide Writing Group. The International Diabetes Federation's guide for diabetes epidemiological studies. Diabetes Res Clin Pract. 2021 Feb;172:108630. [PMID: 33347900]

[3] Ogurtsova K, Guariguata L, Barengo NC, Ruiz PL, Sacre JW, Karuranga S, Sun H, Boyko EJ, Magliano DJ. IDF diabetes Atlas: Global estimates of undiagnosed diabetes in adults for 2021. Diabetes Res Clin Pract. 2022 Jan;183:109118. [PMID: 34883189]

[4] Shah N, Abdalla MA, Deshmukh H, Sathyapalan T. Therapeutics for type-2 diabetes mellitus: a glance at the recent inclusions and novel agents under development for use in clinical practice. Ther Adv Endocrinol Metab. 2021 Sep 23;12:20420188211042145. [PMID: 34589201]

[5] Yendapally R, Sikazwe D, Kim SS, Ramsinghani S, Fraser-Spears R, Witte AP, La-Viola B. A review of phenformin, metformin, and imeglimin. Drug Dev Res. 2020 Jun;81(4):390-401. [PMID: 31916629]

[6] Dubourg J, Ueki K, Grouin JM, Fouqueray P. Efficacy and safety of imeglimin in Japanese patients with type 2 diabetes: A 24-week, randomized, doubleblind, placebo-controlled, dose-ranging phase $2 \mathrm{~b}$ trial. Diabetes Obes Metab. 2021 Mar;23(3):800-10. [PMID: 33275318]

[7] Abdelhaleem IA, Salamah HM, Alsabbagh FA, Eid AM, Hussien HM, Mohamed NI, Ebada MA. Efficacy and safety of imeglimin in patients with type 2 diabetes mellitus: A systematic review and metaanalysis of randomized clinical trials. Diabetes Metab Syndr. 2021 Nov-Dec;15(6):102323. [PMID: 34717136] [8] Fouqueray P, Pirags V, Diamant M, Schernthaner G, Lebovitz HE, Inzucchi SE, Bailey CJ. The efficacy and safety of imeglimin as add-on therapy in patients with type 2 diabetes inadequately controlled with sitagliptin 
Citation: Okada M, Bando H, Iwatsuki N, Ogawa T, Sakamoto K. Clinical Efficacy of Imeglimin (Twymeeg) for Elderly Patient with Type 2 Diabetes Mellitus (T2DM). Asp Biomed Clin Case Rep. 2022 Feb 21;5(1):33-37.

Case Report

monotherapy. Diabetes Care. 2014 Jul;37(7):1924-30.

[PMID: 24722500]

[9] Giruzzi M. Imeglimin. Clin Diabetes. 2021 Oct;39(4):439-40. [PMID: 34866787]

[10] Pirags V, Lebovitz H, Fouqueray P. Imeglimin, a novel glimin oral antidiabetic, exhibits a good efficacy and safety profile in type 2 diabetic patients. Diabetes Obes Metab. 2012 Sep;14(9):852-58. [PMID: 22519919] [11] Funazaki S, Yoshida M, Yamada H, Kakei M, Kawakami M, Nagashima S, Hara K, Dezaki K. A novel mechanism of imeglimin-mediated insulin secretion via the cADPR-TRP channel pathway. J Diabetes Investig. 2022 Jan;13(1):34-41. [PMID: 34523242]

[12] Hallakou-Bozec S, Vial G, Kergoat M, Fouqueray P, Bolze S, Borel AL, Fontaine E, Moller DE. Mechanism of action of Imeglimin: A novel therapeutic agent for type 2 diabetes. Diabetes Obes Metab. 2021 Mar;23(3):664-73. [PMID: 33269554]

[13] Dubourg J, Fouqueray P, Quinslot D, Grouin JM, Kaku K. Long-term safety and efficacy of imeglimin as monotherapy or in combination with existing antidiabetic agents in Japanese patients with type 2 diabetes (TIMES 2): A 52-week, open-label, multicentre phase 3 trial. Diabetes Obes Metab. 2021 Dec 6. Epub ahead of print. [PMID: 34866306]

[14] Tomita Y, Hansson E, Mazuir F, Wellhagen GJ, Ooi QX, Mezzalana E, Kitamura A, Nemoto D, Bolze S. Imeglimin population pharmacokinetics and dose adjustment predictions for renal impairment in Japanese and Western patients with type 2 diabetes. Clin Transl Sci. 2021 Dec 27. Epub ahead of print. [PMID: 34962074] 